Cite this: Photochem. Photobiol. Sci., 2013, 12, 1202

Received 23rd January 2013,

Accepted 25th March 2013

DOI: $10.1039 / c 3 p p 50020 a$

www.rsc.org/pps

\section{The photochemical ring opening reaction of chromene as seen by transient absorption and fluorescence spectroscopy $\dagger$}

\begin{abstract}
Teja T. Herzog, ${ }^{a, b}$ Gerald Ryseck, a,b,c Evelyn Ploetz ${ }^{a, b, c, d}$ and Thorben Cordes ${ }^{\star a, b, d}$
In this paper we investigate the photochromic ring-opening reaction of 2,2-diphenyl-5,6-benzo( $2 \mathrm{H}$ )chromene. In particular, we study the uncertainties and contradictions in various published reaction models using a combination of transient absorption and fluorescence spectroscopy with femtosecond time resolution. We propose a simplified reaction scheme which is in good agreement with theoretical studies. Here, photoexcitation populates a Franck-Condon state, whose fast vibrational wave packet motion, vibrational relaxation, bond-alternation and/or solvent rearrangement processes occur on the sub-picosecond timescale. Our data suggest that the resulting excited state minimum with picosecond lifetime still features structural characteristics of the closed form. Subsequently, the ring-opened photoproducts are formed in a concerted step from the excited state. The velocity of the photoreaction hence only depends on the time that the molecule needs to reach the transition region between the ground and excited states where the crucial bond breakage occurs.
\end{abstract}

\section{Introduction}

Simple chemical transformations, such as pericyclic reactions $^{1,2}$ or $Z / E$-isomerizations, ${ }^{3}$ are the functional basis for a variety of photochromic compounds. ${ }^{4,5}$ Due to their promising applications in both the material and life sciences, the interest in such materials has notably increased over the past few decades. ${ }^{6-8}$ A more fundamental interest is triggered by the fact that photochromic reactions can be studied with extremely high time-resolution down to the femtosecond regime. This fact allows real-time observation of fundamental chemical processes, e.g., bond rotation, bond-formation or cleavage, and also allows a detailed comparison with quantum-chemical calculations. $^{9}$

Naphthopyrans or chromenes (see Scheme 1 for the compound used in the present study, i.e., 2,2-diphenyl-5,6-benzo-

\footnotetext{
${ }^{a}$ Chair for Biomolecular Optics, Faculty of Physics, Ludwig-Maximilians-Universität München, Oettingenstr. 67, D-80538 München, Germany

${ }^{b}$ Munich Center for Integrated Protein Science (CIPSM), München, Germany ${ }^{c}$ Femtosecond Spectroscopy Research Group, Institute for Physical Chemistry II, Heinrich-Heine-Universität Düsseldorf, Universitätsstr. 1, D-40225 Düsseldorf, Germany

${ }^{d}$ Molecular Microscopy Research Group and Single-Molecule Biophysics, Zernike Institute for Advanced Materials, University of Groningen, Nijenborgh 4, 9747 AG Groningen, The Netherlands. E-mail:t.m.cordes@rug.nl; Fax: (+31) 503639199 $\dagger$ Electronic supplementary information (ESI) available: Additional transient absorption and fluorescence data for different excitation and solvent conditions. See DOI: $10.1039 / \mathrm{c} 3 \mathrm{pp} 50020 \mathrm{a}$
}

(2H)chromene, DPBC) belong to the class of photochromic molecules. ${ }^{4,5,10,11}$ The closed form of chromene molecules (CF, Scheme 1) shows only strong absorption in the UV-range due to poor overlap of the $\pi$-electron systems of the different chromophore parts (i.e., the phenyl and pyrane moieties). Irradiation with UV-light initiates a pericyclic ring-opening reaction from $\mathrm{CF}$ to the open form, OF (Scheme 1), which features strong absorption in the green/blue part of the visible spectrum. $^{12}$

The photochemical quantum yield of the ring-opening is close to unity $\left(\Phi_{\mathrm{pc}}=0.7-0.9^{13}\right)$ depending on solvent and excitation conditions. ${ }^{13,14}$ Two stable product isomers $\left(\mathrm{OF}_{(\mathrm{tc})}\right.$, $\mathrm{OF}_{(\mathrm{tt})}$, Scheme 1) could be identified that have nearly identical absorption spectra but differing oscillator strength. ${ }^{12,13,15}$ The ring-closure reaction, i.e., the transformation $\mathrm{OF} \rightarrow \mathrm{CF}$, occurs thermally on various timescales ranging from microseconds to
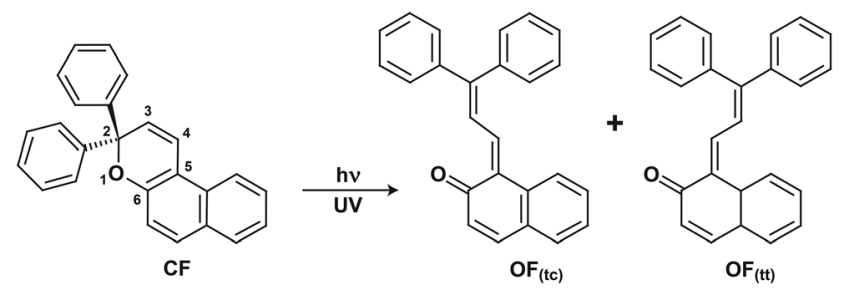

Scheme 1 Schematic representation of the photochemical ring-opening of 2,2-dimethyl-5,6-benzo(2H)chromene (DPBC). Left: chemical structure of the closed form (CF). Right: chemical structure of the open product forms with trans-cis, $\mathrm{OF}_{(\mathrm{tc})}$, and trans-trans, $\mathrm{OF}_{(\mathrm{tt})}$, configuration. 
seconds depending on the specific substitution ${ }^{16,17}$ of the chromene and is believed to originate only from $\mathrm{OF}_{(\mathrm{tc})} \cdot{ }^{12,13}$

A large number of experimental and theoretical studies are currently available $\mathrm{e}^{13,14,16,18-21}$ concentrating on the molecule DPBC (Scheme 1) or related compounds. These compounds were investigated in different solvents (polar vs. non-polar) ${ }^{13}$ and for different excitation conditions, $\left(\mathrm{S}_{1} v s . \mathrm{S}_{N} \text { excitation }\right)^{19}$ using continuous-wave spectroscopy ${ }^{12,13,15,22,23}$ or timeresolved techniques, e.g., transient absorption, TA. ${ }^{14,18-20,24,25}$

Based on the data from transient absorption measurements and the spectral dynamics observed after photoexcitation, at first a sequential model with fast excited state decay was suggested for the ring-opening reaction (Fig. 1a). ${ }^{14,18-20}$

In this model, it was hypothesized that photoexcitation to the Franck-Condon range leads to ultrafast bond-cleavage (Scheme 1, $\mathrm{O}_{1}-\mathrm{C}_{2}$ ), i.e., the formation of an open-state precursor in the excited state (Fig. 1a, Model with fast excited state decay) on a time-scale faster than the actual time-resolution ( $\sim 150 \mathrm{fs}$ ) of the experiment. It should be mentioned that up to now there has been no experimental evidence to support the hypothesis for ultrafast formation of a zwitter-ionic or biradicaloid open-state. ${ }^{14,18-20}$ The first directly observable process in the TA-data is a rapid decay $\left(<0.5 \mathrm{ps}, \tau_{1}\right)$ of a broad and unstructured induced absorption, an observation that was interpreted as decay of the excited state and population of a "cisoid" ground-state conformation $\mathrm{S}_{0}\left(\mathrm{OF}_{\text {cis }}\right)$ of DPBC (Fig. 1a). ${ }^{14,18-20}$ Subsequently, a prominent build-up of absorption in the spectral region of $\mathrm{OF}_{(\mathrm{tc})}$ and $\mathrm{OF}_{(\mathrm{tt})}$ with time constant $\tau_{2}$ of the order of picoseconds was assigned to singlebond rotation around $\mathrm{C}_{3}-\mathrm{C}_{4}$ (Scheme 1) and hence formation of product isomers. Finally, smaller changes of the induced absorption on timescales $>10$ ps $\left(\tau_{3}\right)$ were assigned to thermal equilibration between product isomers $\mathrm{OF}_{(\mathrm{tc})}$ and $\mathrm{OF}_{(\mathrm{tt})}$.

As realized in additional experiments with DPBC and other substances, the use of transient absorption was not sufficient to univocally assign the rapid decay of absorption in the early time-regime to the decay of the excited singlet-state. ${ }^{14,20}$ As there was no clear signature of an excited state in the TA-experiments (i.e., stimulated emission) it remained uncertain which time constants were actually linked to the excited and hence fluorescing states. This resulted in the postulation of a second potential model for the ring-opening of DPBC involving a longer-lived excited state with otherwise identical follow-up processes (Fig. 1b, Model with excited state minimum). ${ }^{14,20}$

In the meantime, a contradicting reaction model (Fig. 1c, Branched model) was proposed by Görner and Chibisov ${ }^{13}$ that was based on data from continuous-wave absorption spectroscopy. Here, a branching into two different excited state conformers is suggested. After excitation the excited state of CF branches into two different excited states, which decay into the latter products $\mathrm{OF}_{(\mathrm{tc})}$ and $\mathrm{OF}_{(\mathrm{tt})}$.

In this paper, we aim to clarify outstanding questions and contradictions assuming that each of the suggested models in Fig. 1 would have a distinct (combined) signature in time-resolved absorption and fluorescence experiments. We hence recorded this combined signature by conducting an
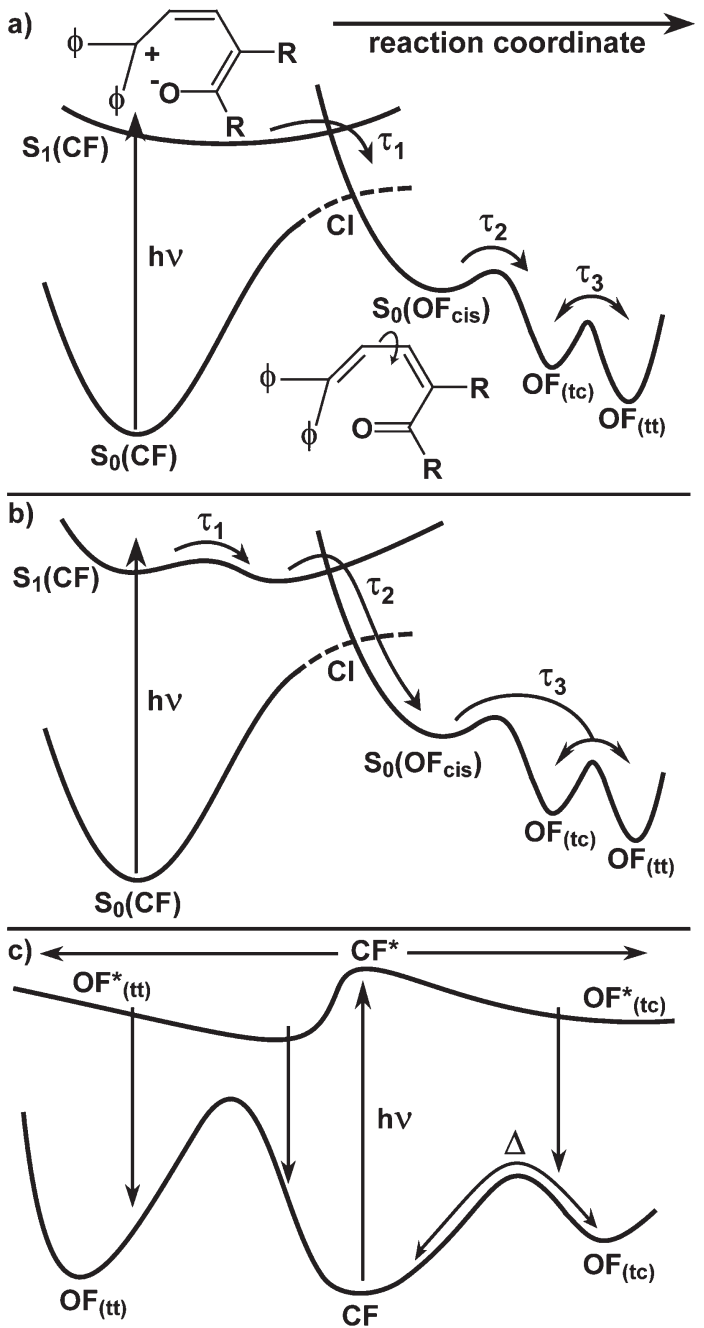

Fig. 1 Schematic representation of proposed models for the ring-opening of DPBC: (a) Model with fast excited-state decay: a barrierless relaxation with time constant $\tau_{1}$ leads to a ground state open cisoid intermediate, $\mathrm{S}_{0}\left(\mathrm{OF}_{\text {cis }}\right)$. Subsequently the photoproduct $\mathrm{OF}_{(\mathrm{tc})}$ is formed with time constant $\tau_{2}$ followed by thermal equilibration between both product isomers $\mathrm{OF}_{(\mathrm{tc})}$ and $\mathrm{OF}_{(\mathrm{tt})}$ with time constant $\tau_{3}$. Model adapted from ref. 14, 18-20. (b) Model with excited-state minimum: a similar model as that described under (a) with an excited-state minimum that is reached from the Franck-Condon region with $\tau_{1}$. In order to form the ground state open cisoid intermediate $S_{0}\left(O F_{c i s}\right)$ a small barrier needs to be overcome corresponding to $\tau_{2}$. Model adapted from ref. 14 and 20. (c) Branched Model: a branched reaction scheme, which suggests that the ringopening takes place immediately after photo-excitation. A branching into the differing excited states of both isomers occurs and is followed by relaxation to the product ground states $\mathrm{OF}_{(\mathrm{tc})}$ and $\mathrm{OF}_{(\mathrm{tt})}$. Model adapted from ref. 13.

experimental study of the parent compound DPBC using a combination of transient absorption (TA) and transient fluorescence spectroscopy (TFl) with femtosecond time resolution.

Our central experiments of DPBC were carried out in the polar solvent acetonitrile. In TA we observe an identical behaviour as reported earlier, ${ }^{14,18-20}$ i.e., the occurrence of a sub-picosecond time-constant (broad featureless absorption decay), a picosecond component (rise of product absorption) and a component on the scale of several tens of ps showing a cooling signature. Interestingly, TFl experiments clearly show a 
biphasic decay of the DPBC emission. We find that the decay of the emission is identical to the picosecond time-constant which is associated with the formation of the product isomers $\mathrm{OF}_{(\mathrm{tc})}$ and $\mathrm{OF}_{(\mathrm{tt})}$. Finally, we compare similar transient data of DPBC for different solvents, viscosity and excitation conditions to provide a comprehensive insight into the ring-opening reaction.

Our experimental findings suggest a simple sequential model with significant differences to all formerly proposed schemes: The molecule undergoes fast intramolecular vibrational relaxation and bond-alternation $(\approx 0.2 \mathrm{ps})$ after photoexcitation to the Franck-Condon region and thereby populates an excited state minimum with a chemical structure that is nearly unaltered with respect to the closed form. Subsequently, the molecule has to reach a transition region (CoIn) in between the ground and excited states which allows branching into educt and product isomers. This picosecond process is slightly activated but shows hardly any viscosity dependence. Our results are further fully consistent with quantum chemical calculations which suggest that the ring-opening process (i.e., bond-cleavage between $\mathrm{O}_{1}-\mathrm{C}_{2}$, compare Scheme 1) is actually to occur at the crossing point between $S_{1} / S_{0}$ and not in the excited state. ${ }^{17,26,27}$

\section{Materials and methods}

\section{Reagents}

Unless otherwise mentioned, solvents (acetonitrile, hexane, dodecane) of spectroscopic grade were used. All experiments were conducted at room temperature. 2,2-Diphenyl-5,6-benzo(2H)chromene, DPBC, was purchased from TCI Europe (product code D3197; CAS 4222-20-2) and used as received without further purification (purity $>98 \%$, LC).

\section{Stationary spectroscopy}

Steady-state absorption spectra in the UV/Vis were recorded using a Perkin-Elmer Lambda 750 spectrophotometer. The closed isomer was illuminated inside a $10 \mathrm{~mm}$ QS cell (Hellma) with a mercury-xenon lamp (Hamamatsu Lightningcure LC4-L8444) in combination with a $2 \mathrm{~mm}$ filter UG1 (Schott) with a strong emission peak at $365 \mathrm{~nm}$ yielding sample PSS after 29 minutes of irradiation and constant stirring of the solution.

\section{Transient absorption spectroscopy in the UV/Vis}

The apparatus for time-resolved absorption experiments has been described elsewhere. ${ }^{9}$ In brief, femtosecond pump pulses were used in combination with suitably delayed probing pulses at different wavelengths. ${ }^{28,29}$ They are based on a Tisapphire laser-amplifier system (Spitfire Pro, Spectra Physics) operated at a central wavelength of $\sim 800 \mathrm{~nm}$ and pulse durations of $\sim 100 \mathrm{fs}$ and a repetition rate of $1 \mathrm{kHz}$. The excitation (pump) pulses in the near UV at $266 \mathrm{~nm}$ and $325 \mathrm{~nm}$ were generated using the frequency doubled output of a twostage non-collinear optical parametric amplifier (NOPA). ${ }^{30,31}$
In the experiment every second pump pulse was blocked by a mechanical chopper in order to improve referencing when recording the pump-induced absorption changes of the sample. The sample solutions were investigated in a flow cell and continuously exchanged by a peristaltic pump at flow rates sufficient to exchange the sample volume (flow-cell with fusedsilica windows, front $0.2 \mathrm{~mm}$, back $1.0 \mathrm{~mm}$ and a channel of $1.0 \mathrm{~mm}$ diameter) in the laser focus between two consecutive laser pulses.

The pump pulses in the UV/Vis experiments had an excitation energy of $\sim 200 \mathrm{~nJ}$ (beam diameter in the sample $d_{\text {pump }}$ $=170 \mu \mathrm{m})$. Probing of the induced absorption changes was performed by using a suitably delayed white-light continuum generated in $\mathrm{CaF}_{2}(\sim 350-700 \mathrm{~nm}$, beam diameter in the sample $\left.d_{\text {probe }}=60 \mu \mathrm{m}\right)$ with the polarization between the two beams at the magic angle.

The spectrum of the transmitted probing light was measured by using a multi-channel detection system: The probe pulses were dispersed by a concave grating (Zeiss, focal distance at $200 \mathrm{~nm}$ is $111 \mathrm{~mm}$, groove number is $320 \mathrm{~mm}^{-1}$, blazed at $225 \mathrm{~nm}$ ) on a NMOS linear image sensor (a Hamamatsu 14-S3902-512Q in combination with a Tec5 DZA-S3901-4 1M preamplifier electronic).

The data points at certain delay times between pump and probe pulses were measured with 4 repetitive scans and with 8000 laser shots per data point. Transient background signals from the pure solvent were weighted and subtracted from the sample signal. The delay time zero of all individual probe wavelengths was determined by a Sellmeier fit. The experimental response time was $\sim 200$ fs (cross-correlation between the pump and the probe).

\section{Transient fluorescence spectroscopy}

Broadband femtosecond-time-resolved fluorescence spectra were collected using a Kerr shutter (Kerr gate) setup detailed elsewhere. ${ }^{32}$ Briefly, sample solutions were excited with laser pulses at $320 \mathrm{~nm}$ focused to a diameter of $\sim 150 \mu \mathrm{m}$. The pulse energies were $\sim 400 \mathrm{~nJ}$ with pulse durations of $\sim 40$ fs. The fluorescence emission was detected in a forward scattering geometry with a nitrogen-cooled CCD camera after passing the Kerr gate and spectral dispersion in a grating spectrograph. The gate pulses were centered at $1060 \mathrm{~nm}$ with a duration of $\sim 50$ fs and energies around $30 \mu \mathrm{J}$. A $1.2 \mathrm{~mm}$ thick fused silica plate served as the Kerr medium. The time resolution of the experiments was $\sim 220$ fs (FWHM of the internal response function), the contrast and efficiency (at the excitation wavelength) of the Kerr shutter amounted to $1: 350$ and $4 \%$ respectively. Scattered light of the gate and excitation beams was suppressed using a combination of a GG 375 and a KG 5 filter. The sample solutions (total volume of $10 \mathrm{ml}$ with a concentration of $\sim 3 \mathrm{mM}$ ) were pumped through a fused silica flow cell ( $1 \mathrm{~mm}$ path length) to avoid multiple excitation. At each setting of the delay between the excitation and gate pulses (total 60 points, 40 points separated by 40 fs starting at $-500 \mathrm{fs}$, logarithmic afterwards) the fluorescence signal was accumulated for 5 seconds and the results of 8 scans of the 
delay line were summed up. Correction for dispersion and wavelength dependent gate efficiency and detector sensitivity were applied according to the procedure described in ref. 32 .

\section{Results}

\section{cw-characterization of the photoreaction}

In order to characterize the spectral changes of 2,2-diphenyl5,6-benzo $(2 H)$ chromene, DPBC after UV-illumination, steadystate absorbance experiments were performed. The absorption of the DPBC CF-chromophore in the spectral region from 250 to $600 \mathrm{~nm}$ shows peaks centred at $350 \mathrm{~nm}, 300 \mathrm{~nm}$ and $<275 \mathrm{~nm}$ (not shown) in the solvent acetonitrile, ACN. All show significant overlap with vibronic structures giving rise to two $(350 \mathrm{~nm})$ and even three distinguishable peaks within the electronic transitions (Fig. 2, solid line).

Upon UV-illumination the overall structure of the absorption spectrum remains similar in the UV-region but reveals a significant difference $>375 \mathrm{~nm}$. Centred at $425 \mathrm{~nm}$ a new peak arises which is assigned to a mixture of $\mathrm{OF}_{(\mathrm{tc})}-\mathrm{OF}_{(\mathrm{tt})}$ isomers; all results are in accordance with published results. ${ }^{12,13,15,22}$

Before ring-opening, the absorption is given by a superposition of two benzene moieties ( $240 \mathrm{~nm}$, not shown) and a napthopyrane group. After the ring-opening reaction a larger system of delocalized $\pi$-electrons extending over both benzene rings and the napthopyrane moiety causes additional absorption in the visible region. Illumination at $365 \mathrm{~nm}$ allows to form a photostationary state (Fig. 2, dashed); the thermal reaction from $\mathrm{OF}_{(\mathrm{tc})}-\mathrm{OF}_{(\mathrm{tt})} \rightarrow \mathrm{CF}$ was found to occur on the timescale of $\sim 10 \mathrm{~s}$ as shown earlier. ${ }^{12,13,15,22} \mathrm{No} \mathrm{cw}$-fluorescence could be detected in agreement with earlier studies on the system. ${ }^{11,13,22}$

\section{Time-resolved experiments}

In the next step we performed measurements with femtosecond time-resolution $(\approx 200 \mathrm{fs})$ using both absorbance and fluorescence as an observable.

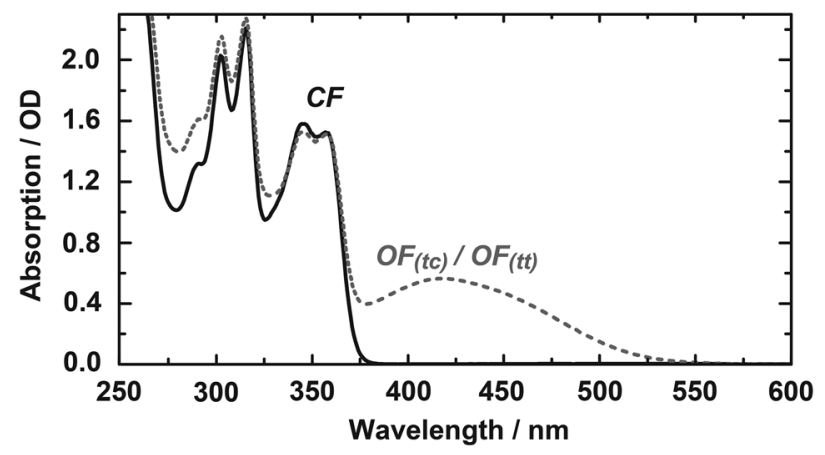

Fig. 2 Absorbance spectra of DPBC in ACN. The spectrum of the closed form (CF) is shown in black. The ring-opening reaction occurs after excitation with UV light $<375 \mathrm{~nm}$. Under continuous UV-illumination CF is transferred into a photostationary state (PSS) which consists of a mixture of $\mathrm{OF}_{(\mathrm{tc})}-\mathrm{OF}(\mathrm{tt})$ photoproducts. The relative amounts of $\mathrm{OF}_{(\mathrm{tc})}-\mathrm{OF}_{(\mathrm{tt})}$ open isomers in the PSS have not been determined.
In transient absorption experiments, photo-excitation of $\mathrm{CF}$ at $325 \mathrm{~nm}$ in the polar solvent ACN results in the ultrafast formation of a broad and nearly featureless induced absorption throughout the complete detection range (Fig. $3 \mathrm{a}, t_{\mathrm{D}}=0.15 \mathrm{ps}$ ) showing only a slight "dip" centered at $425 \mathrm{~nm}$.

As the comparison with Fig. 3c shows (time-resolved fluorescence data), we can assign the reduced signal around $425 \mathrm{~nm}$ (Fig. 3a, $t_{\mathrm{D}}=0.15 \mathrm{ps}$ ) to the red wing of stimulated emission which is superimposed by induced absorption. We find that the broad and featureless induced absorption decays on a subpicosecond timescale (Fig. 3a, compare $t_{\mathrm{D}}=0.15 \mathrm{ps}$ vs. $t_{\mathrm{D}}=$ $1.0 \mathrm{ps}$ ). A prominent absorption band arises at $425 \mathrm{~nm}$ on the picosecond time scale (Fig. 3a, compare $t_{\mathrm{D}}=1.0 \mathrm{ps} v$ s. $t_{\mathrm{D}}=$ $5.0 \mathrm{ps}$ ). Only small changes of the signal are observed on later timescales (Fig. 3a, compare $t_{\mathrm{D}}=5.0 \mathrm{ps}$ vs. $t_{\mathrm{D}}=100.0 \mathrm{ps}$ ) and the spectrum at late delay time $>100 \mathrm{ps}$ resembles the stationary difference between CF and the PSS shown in Fig. 2.

The results from a multi-exponential fit routine (for details see Materials and methods and ref. 33) of the data-set with four components $\tau_{1}, \tau_{2}, \tau_{3}$, and an offset are shown in Fig. 3b in the form of decay-associated spectra (DAS); the quality of the fit can be seen in Fig. $S 2 \dagger$ where chosen time transients for $430 \mathrm{~nm}$ and $600 \mathrm{~nm}$ are plotted for recorded data and the respective fit. We find a fast and mainly positive component with $\tau_{1}=0.2 \pm 0.1 \mathrm{ps}$ that is associated with the decay of a broad and featureless induced absorption (Fig. 3b, black solid line). Build-up of absorption at $425 \mathrm{~nm}$ is found with $\tau_{2}=1.3 \pm$ 0.3 ps (Fig. 3b, blue dash-dotted line). A sigmoidal spectrum with smaller amplitude shows the time constant $\tau_{3}=7 \pm 2$ ps. The difference in absorption between $\mathrm{CF}$ (educt) and $\mathrm{OF}_{(\mathrm{tc})}$ and $\mathrm{OF}_{(\mathrm{tt})}$ (photoproducts) is seen in the offset spectrum (Fig. 3b). These data, i.e., spectra and time constants, are in good agreement with published studies on DPBC. ${ }^{14,18-20}$

In the next step we used a Kerr-gate setup ${ }^{32}$ with similar time-resolution as in the TA-setup $(\approx 200 \mathrm{fs})$ to visualize the fluorescence decay of DPBC. After excitation of CF at $320 \mathrm{~nm}$ we observe an emission spectrum centred around $400 \mathrm{~nm}$ with a tail reaching up to $550 \mathrm{~nm}$ (Fig. $3 \mathrm{c}, t_{\mathrm{D}}=0.15 \mathrm{ps}$ ). The shape of the spectrum is essentially unaltered over the observation period and the signal vanishes within only a few picoseconds (Fig. 3c, compare $t_{\mathrm{D}}=0.15$ ps $v s$. $t_{\mathrm{D}}=3.5 \mathrm{ps}$ ). A similar kinetic analysis as that described for TA-data results in two timeconstants and a negligible offset (Fig. 3d). The biphasic decay of the emission occurs with the time constants $\tau_{1}=0.2 \pm 0.1 \mathrm{ps}$ and $\tau_{2}=1.5 \pm 0.4 \mathrm{ps}$; these results nicely match the time constants obtained by TA and allow the following conclusions: $\tau_{1}$, $\tau_{2}$ are clearly associated with excited electronic states. As $\tau_{2}$ can be assigned to product formation of $\mathrm{OF}_{(\mathrm{tc})}$ and $\mathrm{OF}_{(\mathrm{tt})}$ the photoreaction directly occurs from the excited state.

\section{TA and TFl data from the viewpoint of proposed models}

We are now in a position to compare our data (Fig. 3) with the reaction models proposed in the literature ${ }^{13,14,18-20}$ (Fig. 1). Each reaction model allows us to come up with a distinct hypothesis about the expected TA and TFl data. In detail, TA will report on the timescale of product formation (build-up of 

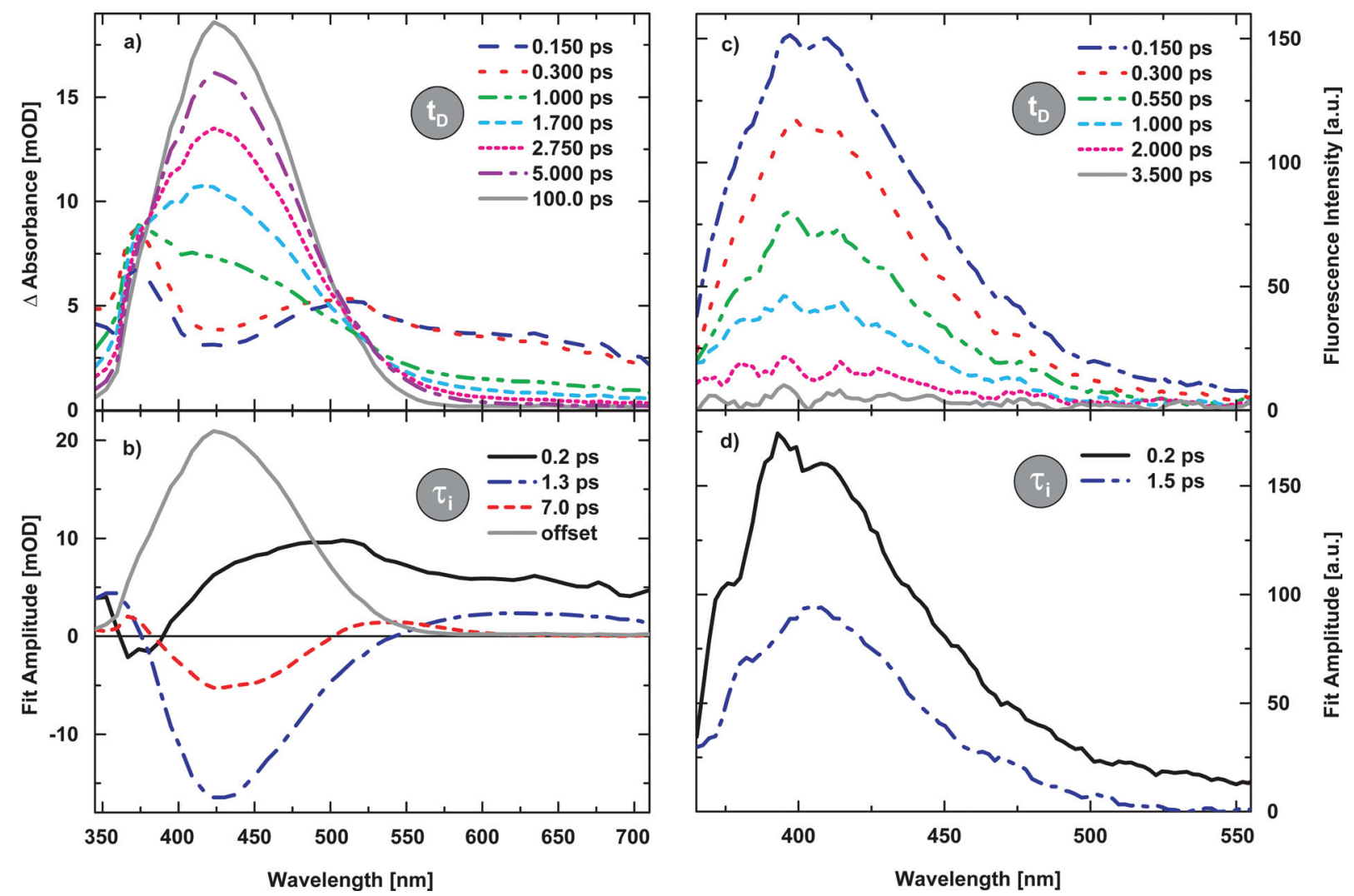

Fig. 3 (a) Time evolution of the absorption spectra following $325 \mathrm{~nm}$ excitation of DPBC closed form in ACN. (b) DAS of the TA experiments resulting from a multiexponential fit analysis with associated time constants $\tau_{i}$. (c) Time evolution of the emission spectra following $320 \mathrm{~nm}$ excitation of DPBC closed form in ACN. (d) DAS of the TFI experiments resulting from a multi-exponential fit analysis with associated time constants $\tau_{i}$

$\mathrm{OF}_{(\mathrm{tc})}$ and $\left.\mathrm{OF}_{(\mathrm{tt})}\right)$ and cooling processes in the ground state, while TFl allows us to assign the time constants and hence processes to excited electronic states.

(a) In the sequential "Model with fast excited-state decay" (Fig. 1a) we expect our TA/TFl data sets to show the following key observations: (i) Product formation, i.e., build-up of absorption at $425 \mathrm{~nm}$, observed by TA, occurs with a picosecond time constant $\left(\tau_{2}\right)$. The excited state decay observed by TFl occurs with only one fast component on the sub-picosecond timescale $\left(\tau_{1}\right)$.

We can clearly rule out a sequential model with fast excited state decay (Fig. 1a) since we were able to show in Fig. 3 that the excited state of DPBC decays bi-exponentially. This is a very strong argument against the proposed sequential model in Fig. 1a. ${ }^{14,18-20}$

We consider branching with $\tau_{1}$ into a cisoid OF and a remaining excited state population unlikely for the following reasons. The quantum yield of the ring-opening process is $\sim 0.8^{13}$ meaning that only a fraction of 0.2 of the population would reside in the excited state. Therefore this small fraction would cause substantial amplitude of the $\tau_{2}$-process observed in the transient emission. This observation would only be possible for an unlikely increase in oscillator strength of emission upon branching out the Franck-Condon range. We consider model (a) to be rather unrealistic and inconsistent with our results from combined TA/TFl experiments. (b) Also the sequential "Model with excited state minimum" (Fig. 1b) shows several inconsistencies in comparison with our data. The model makes the following predictions: (i) The emission should decay bi-exponentially on the picosecond timescale $\left(\tau_{1}, \tau_{2}\right)$ forming a cisoid-precursor in the electronic ground state with the picosecond component. Final product formation occurs on longer timescales $\left(\tau_{3}\right)$.

Although we do observe experimentally that the excited state finally decays with $\tau_{2}$ the remaining predictions are not satisfactory. We cannot find a reasonable explanation for the TA-data, as Fig. $1 \mathrm{~b}$ predicts that a cisoid-precursor is formed with $\tau_{2}$ before the final formation of the photoproduct with time constant $\tau_{3}{ }^{4,20}$ Our data give no spectroscopic evidence for the formation of such a cisoid intermediate on the timescale of $\tau_{2}$. In contrast, we clearly observe that the excited-state decay occurs simultaneously with product formation (i.e., $\tau_{2}$ ) and does not proceed on later timescales, i.e., $\tau_{3}$.

We hence conclude that also model (b) does not describe our experimental observations adequately. As discussed for model (a), branching will not render this model more likely.

(c) We finally consider a purely "Branched model" (Fig. 1c) where the molecules branch faster than our experimental time-resolution of $\sim 200$ fs. This process forms excited-state precursors of the two open-state isomers (Fig. 1c). ${ }^{13}$ We hence expect to observe two different decay components in the emission signal (observed by $\mathrm{TFl}$ ) with simultaneous product 
formation from both time constants (observed by TA). One of the time constants should also be associated with repopulation of the CF-ground-state.

Such a model is fully inconsistent with our data as the formation of photoproduct $\left(\mathrm{OF}_{(\mathrm{tc})}\right.$ or $\left.\mathrm{OF}_{(\mathrm{tt})}\right)$ is only associated with one time constant, i.e., $\tau_{2}$, and not with two time constants.

\section{Refining the model}

Due to the fact that neither of the models in Fig. 1 is able to explain our findings we propose a much simpler reaction scheme as depicted in Fig. 4. After photoexcitation of CF with $320 \mathrm{~nm}$ light either the first or second excited singlet state $\mathrm{S}_{1} / \mathrm{S}_{2}$ is populated in the Franck-Condon region $\mathrm{FC}^{*}$. Subsequently, fast vibrational wave packet motion, vibrational relaxation, bond-alternation and/or solvent rearrangement processes occur that lead to the vibrational ground state of $S_{1}$ with the time constant $\tau_{1}$ (Fig. 4). As the potential energy surface of chromenes was described to be rather flat, ${ }^{17,26,27}$ this process is seen in both TA (as a reduction of induced absorption, Fig. 3b) and in TFl (as a decay of oscillator strength, Fig. 3d). We have, however, no spectroscopic indication that the ring opening (breakage of $\mathrm{O}_{1}-\mathrm{C}_{2}$, see Scheme 1) already occurs within this time window. We base this statement, which is in clear contrast to all models published so far, on two observations: (i) There is no formation of a spectrum that would match such a "cisoid" geometry in TA, i.e., a "blue-shifted" version of the product spectrum; (ii) we also expect that this cisoid state would have a neligible oscillator strength in emission, something that is not in agreement with our observation

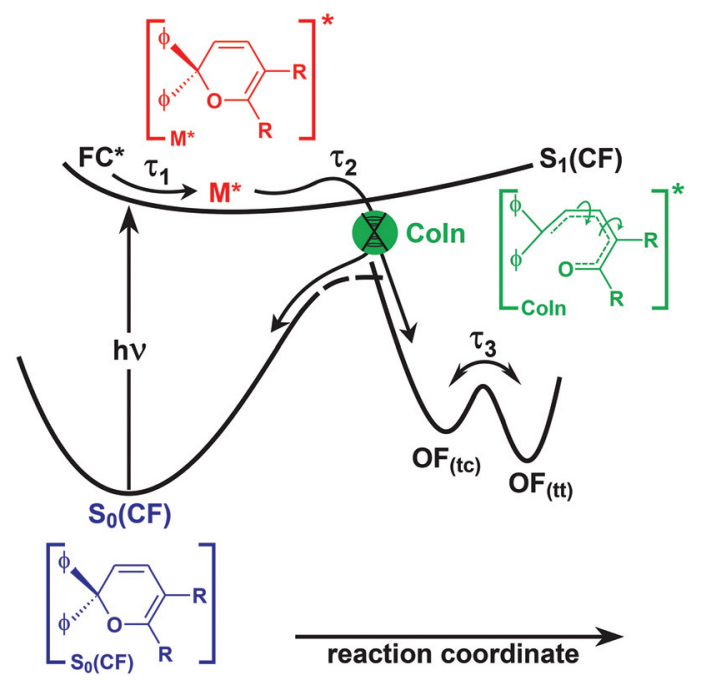

Fig. 4 Proposed reaction scheme of DPBC after excitation by UV light. In a first step relaxation from the Franck-Condon region $\left(F C^{*}\right)$ into an excited state minimum $\left(\mathrm{M}^{*}\right)$ occurs $\left(\tau_{1}\right)$, which is followed by a transition through a conical intersection (Coln, $\left.\tau_{2}\right)$. Here branching of the population into product $(80 \%)^{13}$ and educt (20\%) occurs in ACN; the branching ratio depends on solvent and excitation conditions. Bond cleavage between oxygen and spiro-carbon produces both open form isomers OF. The $\mathrm{OF}_{(\mathrm{tc})}$ isomer in trans-cis geometry can transform into the trans-trans isomer $\mathrm{OF}_{(\mathrm{tt})}$, by rotation around the $\mathrm{C}(4)-\mathrm{C}(5)$ double bond (thermal equilibration, $\tau_{3}$ ). of a second time-constant $\tau_{2}$ with large amplitude in the DAS (Fig. 3d). In agreement with theoretical studies ${ }^{17,26,27}$ we propose that the formed excited state minimum $\mathbf{M}^{*}$ (Fig. 4) has a similar structure to the original closed form $\mathrm{CF}$, with only slightly attenuated bond lengths accompanying electronic excitation.

The transition into the ground state is then hindered by an insignificant activation barrier ${ }^{17,26,27}$ and occurs on the picosecond timescale with time constant $\tau_{2}$. The process is characterized by simultaneous decay of emission and formation of product absorption $\left(\mathrm{OF}_{(\mathrm{tc})}-\mathrm{OF}_{(\mathrm{tt})}\right)$. In agreement with quantum chemical calculations we propose that the actual ring-opening process occurs with this time constant and is a concerted process. At the crossing point between the ground and excited states (Fig. 4, conical intersection, CoIn) the bond between $\mathrm{O}_{1}$ and $\mathrm{C}_{2}$ is finally broken for the majority of the population. The final question now concerns the fate of the ring-opened molecules, i.e., in which of the two possible stereo-isomer states will they end up.

Our TA-data (Fig. 3d) shows that on the timescale of several picoseconds $\left(\tau_{3}\right)$ there are two processes occuring: (i) non-exponential cooling where the product emission shifts from longer wavelength (vibrationally "hot") to shorter ones (relaxed). This is seen as a sigmoidal shaped feature in the DAS (Fig. 3d, $6.5 \mathrm{ps}$, positive amplitude of $525 \mathrm{~nm}$ and negative amplitude of $425 \mathrm{~nm}$ ). (ii) Additionally there is a strong negative contribution at $425 \mathrm{~nm}$ that is not associated with cooling. Since the negative contribution exceeds the positive one we interpret this as an overlaying population transfer between the two product isomers due to the fact that both product isomers are believed to have essentially identical absorption spectra but differing oscillator strength. ${ }^{12}$ Unfortunately, we cannot answer the question whether initially (after $\tau_{2}$ ) only one or both products have been formed.

Finally it should be noted that we find nearly identical results in the non-polar solvent hexane (see Fig. S1 $\dagger$ ) suggesting the same model for different solvents.

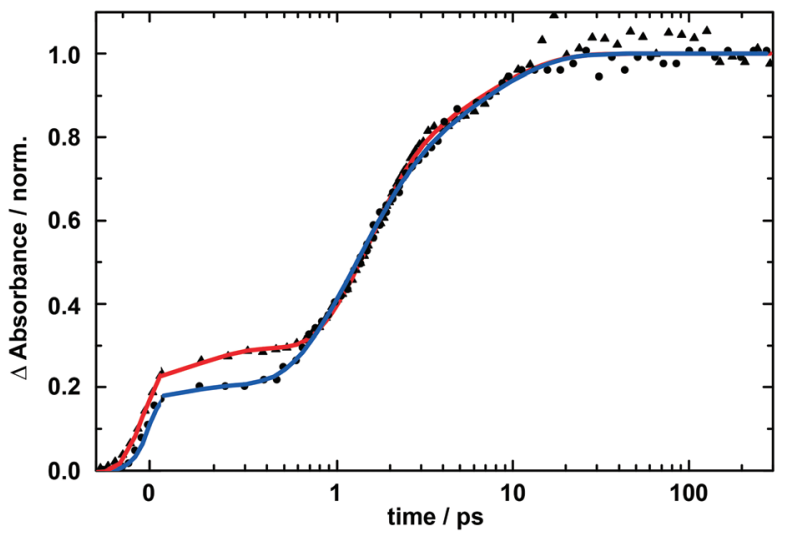

Fig. 5 Transient absorption signal of DPBC in hexane (dotted data points, blue fit curve) and dodecane (triangle data points, red fit curve) after $266 \mathrm{~nm}$ photo excitation, probed at $430 \mathrm{~nm}$ and normalized to the value at $200 \mathrm{ps}$. The time constants for the multi-exponential fit function are summarized in Table 1. Complete data sets of the experiments are shown in Fig. S3.† 
Table 1 Summary of time constants derived from a multi-exponential fit routine of pump-probe data of DPBC under different solvent and excitation conditions

\begin{tabular}{llllr}
\hline Method & $\lambda_{\text {exc }}$ & Solvent & $\tau_{1}$ & $\tau_{2}$ \\
\hline TA & $325 \mathrm{~nm}$ & ACN & $0.20 \pm 0.10 \mathrm{ps}$ & $1.3 \pm 0.3 \mathrm{ps}$ \\
TFl & $320 \mathrm{~nm}$ & ACN & $0.20 \pm 0.15 \mathrm{ps}$ & $1.5 \pm 0.4 \mathrm{ps}$ \\
TA & $325 \mathrm{~nm}$ & Hexane & $0.20 \pm 0.10 \mathrm{ps}$ & $1.3 \pm 0.3 \mathrm{ps}$ \\
TFl & $320 \mathrm{~nm}$ & Hexane & $0.20 \pm 0.15 \mathrm{ps}$ & $1.2 \pm 0.2 \mathrm{ps}$ \\
TA & $266 \mathrm{~nm}$ & Hexane & $0.35 \pm 0.12 \mathrm{ps}$ & $1.2 \pm 0.4 \mathrm{ps}$ \\
TA & $266 \mathrm{~nm}$ & Dodecane & $0.35 \pm 0.12 \mathrm{ps}$ & $9 \pm 0.3 \mathrm{ps}$ \\
& & & & $1.0 \pm 0.3 \pm 2 \mathrm{ps}$ \\
\hline
\end{tabular}

According to our model it is also sufficient to overcome the barrier in $S_{1}$ to reach the CoIn and hence the electronic ground state. As this process does not require major structural rearrangements within the molecule that could cause a viscosity dependence we finally aim to validate our model. For that we performed experiments to demonstrate that the ratelimiting step of the reaction (i.e., $\tau_{2}$ ) is not substantially affected by changes in viscosity. In Fig. 5 we show normalized TA time-transients of DPBC in solvents of different viscosity.

The two chosen transients monitor the formation of the photoproducts. Inspection of the graph shows that there is essentially no difference between a solvent of low viscosity (Fig. 5, blue curve, hexane) and high viscosity (Fig. 5, red curve, dodecane). The results from a global fit routine are summarized in Table 1 with those from data in Fig. 3/S1. $\dagger$

From this we can state that the formation of the photoproduct, which is characterized by the time constant $\tau_{2}$, is essentially independent of solvent, viscosity and even excitation conditions; compare the results from a global fitting routine summarized in Table 1. Excitation to higher excited states only moderately alters the initial excited state propagation $\left(\tau_{1}\right)$.

\section{Conclusions}

In summary, we reported on the re-evaluation of formerly proposed models ${ }^{13,14,17-20,26,27}$ for the photoinduced ring-opening reaction of the chromene compound 2,2-diphenyl-5,6-benzo$(2 H)$ chromene, DPBC. To clarify uncertainties and contradictions in various reaction models ${ }^{13,14,18-20}$ we used a combination of time-resolved spectroscopy, i.e., transient absorption and transient fluorescence spectroscopy with sub-picosecond time resolution. None of the published models adequately describes our experimental findings from combined absorption and emission experiments. We hence proposed a new and simple reaction scheme which is in good agreement with theoretical studies on the same subject. ${ }^{17,26,27}$ Here, the velocity of the photoreaction of DPBC solely depends on the time that the molecule needs to reach a conical intersection where the crucial bond between carbon and oxygen is finally broken. So, our study has implications for the understanding of chromene photochemistry, reaction mechanisms of compounds with related photochemistry and consequently also for the design of photochromic materials with custom-made properties.

\section{Acknowledgements}

We thank W. Zinth for generous support, discussions and the possibility to perform this study within his laboratory facilities. The study was financed by the DFG through SFB749. T. Cordes was additionally supported by the Centre for NanoScience (CeNS, LMU München), the Zernike Institute for Advanced Materials (University of Groningen) and the Centre of Synthetic Biology (University of Groningen).

\section{References}

1 M. Irie, Chem. Rev., 2000, 100, 1685-1716.

2 N. Tamai and H. Miyasaka, Chem. Rev., 2000, 100, 18751890.

3 C. Dugave and L. Demange, Chem. Rev., 2003, 103, 24752532.

4 J. C. Crano and R. J. Guglielmetti, Organic photochromic and thermochromic compounds - Volume 1: Main photochromic families - Introduction, Plenum Press Div Plenum Publishing Corp., New York, 1999.

5 H. Dürr, Angew. Chem., 2004, 116, 3404-3418.

6 D. L. Fortin, M. R. Banghart, T. W. Dunn, K. Borges, D. A. Wagenaar, Q. Gaudry, M. H. Karakossian, T. S. Otis, W. B. Kristan, D. Trauner and R. H. Kramer, Nat. Methods, 2008, 5, 331-338.

7 M. Irie, T. Fukaminato, T. Sasaki, N. Tamai and T. Kawai, Nature, 2002, 420, 759-760.

8 S. W. Hell, Science, 2007, 316, 1153-1158.

9 (a) A. Nenov, T. Cordes, T. T. Herzog, W. Zinth and R. de VivieRiedle, J. Phys. Chem. A, 2010, 114, 13016-13030; (b) T. Cordes, T. Schadendorf, B. Priewisch, K. Ruck-Braun and W. Zinth, J. Phys. Chem. A, 2008, 112, 581-588; (c) T. Cordes, T. Schadendorf, K. Ruck-Braun and W. Zinth, Chem. Phys. Lett., 2008, 455, 197-201.

10 R. S. Becker and J. Kolc, J. Phys. Chem., 1968, 72, 997-1001.

11 C. Lenoble and R. S. Becker, J. Photochem., 1986, 33, 187197.

12 G. Ottavi, G. Favaro and V. Malatesta, J. Photochem. Photobiol., A, 1998, 115, 123-128.

13 H. Görner and A. K. Chibisov, J. Photochem. Photobiol., A, 2002, 149, 83-89.

14 B. Moine, J. Rehault, S. Aloise, J. C. Micheau, C. Moustrou, A. Samat, O. Poizat and G. Buntinx, J. Phys. Chem. A, 2008, 112, 4719-4726. 
15 S. Delbaere, B. Luccioni-Houze, C. Bochu, Y. Teral, M. Campredon and G. Vermeersch, J. Chem. Soc., Perkin Trans. 2, 1998, 1153-1157.

16 F. Ortica, P. Smimmo, G. Favaro, U. Mazzucato, S. Delbaere, D. Venec, G. Vermeersch, M. Frigoli, M. C. Corinne and A. Samat, Photochem. Photobiol. Sci., 2004, 3, 878-885.

17 F. Zerbetto, S. Monti and G. Orlandi, J. Chem. Soc., Faraday Trans. 2, 1984, 80, 1513-1527.

18 J. Aubard, F. Maurel, G. Buntinx, O. Poizat, G. Levi, R. Guglielmetti and A. Samat, Mol. Cryst. Liq. Cryst., 2000, 345, 539-544.

19 P. L. Gentili, E. Danilov, F. Ortica, M. A. J. Rodgers and G. Favaro, Photochem. Photobiol. Sci., 2004, 3, 886-891.

20 B. Moine, G. Buntinx, O. Poizat, J. Rehault, C. Moustrou and A. Samat, J. Phys. Org. Chem., 2007, 20, 936-943.

21 J. Hobley, V. Malatesta, K. Hatanaka, S. Kajimoto, S. L. Williams and H. Fukumura, Phys. Chem. Chem. Phys., 2002, 4, 180-184.

22 S. Jockusch, N. J. Turro and F. R. Blackburn, J. Phys. Chem. A, 2002, 106, 9236-9241.

23 N. Rebiere, C. Moustrou, M. Meyer, A. Samat, R. Guglielmetti, J. C. Micheau and J. Aubard, J. Phys. Org. Chem., 2000, 13, 523-530.
24 C. Bottcher, G. Zeyat, S. A. Ahmed, E. Irran, T. Cordes, C. Elsner, W. Zinth and K. Rueck-Braun, Beilstein J. Org. Chem., 2009, 5.

25 Y. Kodama, T. Nakabayashi, K. Segawa, E. Hattori, M. Sakuragi, N. Nishi and H. Sakuragi, J. Phys. Chem. A, 2000, 104, 11478-11485.

26 P. N. Day, Z. Q. Wang and R. Pachter, J. Phys. Chem., 1995, 99, 9730-9738.

27 A. Migani, P. L. Gentili, F. Negri, M. Olivucci, A. Romani, G. Favaro and R. S. Becker, J. Phys. Chem. A, 2005, 109, 8684-8692.

28 R. Huber, H. Satzger, W. Zinth and J. Wachtveitl, Opt. Commun., 2001, 194, 443-448.

29 M. Seel, E. Wildermuth and W. Zinth, Meas. Sci. Technol., 1997, 8, 449-452.

30 E. Riedle, M. Beutter, S. Lochbrunner, J. Piel, S. Schenkl, S. Sporlein and W. Zinth, Appl. Phys. B: Lasers Opt., 2000, 71, 457-465.

31 T. Wilhelm, J. Piel and E. Riedle, Opt. Lett., 1997, 22, 14941496.

32 B. Schmidt, S. Laimgruber, W. Zinth and P. Gilch, Appl. Phys. B: Lasers Opt., 2003, 76, 809-814.

33 H. Satzger and W. Zinth, Chem. Phys., 2003, 295, 287295. 